\title{
APPLICATION OF KALMAN AND RLS ADAPTIVE ALGORITHMS TO NON-LINEAR LOUDSPEAKER CONTROLER PARAMETER ESTIMATION: A CASE STUDY
}

\author{
Ricardo Adriano Ribeiro ${ }^{(1,2)}$, António Joaquim Serralheiro ${ }^{(1,3)}$, Moisés Simões Piedade $^{(1,2,3)}$ \\ (1) INESC-ID - Rua Alves Redol 9 Apartado 13069 1000-029 Lisboa Portugal \\ ${ }^{(2)}$ Instituto Superior Técnico - Av. Rovisco Pais, 1049-001 Lisboa Portugal \\ ${ }^{(3)}$ Academia Militar - Rua Gomes Freire, 1150-175 Lisboa Portugal
}

\begin{abstract}
The loudspeaker is a nonlinear transducer that produces harmonic distortion and nonlinear controllers, requiring parameters well tuned to the loudspeaker, are used to reduce it. Unfortunately loudspeaker parameters are not well known and vary during normal operation. Based on a simplified nonlinear model of the loudspeaker, and a modification to the adaptive filters, nonlinear systems for the estimation of parameter for the mechanical part and for the electrical part of the loudspeaker where developed. The parameters estimated are directly usable by the controllers, not requiring additional conversion calculations. The Kalman and RLS adaptive algorithms where applied to this systems and simulation results show that they converge, although the electrical part estimation system was about 15 times slower than the mechanical.
\end{abstract}

\section{INTRODUCTION}

Loudspeakers $(L S)$ are electroacoustic transducers used in reproduction of sound and, unfortunately, they are highly nonlinear [1, $2,3]$. Non-linearities are responsible for the production of harmonic distortion, among other problems. Although these problems do occur in all the perceptible spectrum, in this paper we shall only address the lower part of it, where the $L S$ has to move larger volumes of air causing greater distortion. A common approach to extend the output of the $L S$ to the low frequencies is to increase the volume of the enclosure [4]. However, practical considerations and usability impose restrictions on its size. Also, a smaller size also means a smaller volume of air that has to be compressed within the enclosure and, because of the highly non-linear relationship between the volume of air and the force it applies to the $L S$ diaphragm [3], results in more output distortion. To further reduce this distortion, nonlinear controllers are used $[1,3,5]$, although, they require a large set of parameters well tuned to the controlled $L S$. Unfortunately, due to manufacturing tolerances, thermal variations during operation, aging and other causes, $L S$ parameter values are not well known, especially the nonlinear ones, that are usually not specified by the manufacturer. Thus, an accurate determination of the parameters of the $L S$ (even during normal operation) is mandatory to the good performance of the controller. Systems able to estimate such parameters, preferably during the normal operation of the $L S$, therefore not requiring special test signals or adding mass to the cone (for example) are highly desirable. The approach followed here is to use adaptive algorithms, namely the Kalman and the Recursive Least Squares $(R L S)$ algorithms [6], applied to the nonlinear models of the $L S$, in order to estimate an adequate controller oriented parameters. The models are presented in a way that favors the direct estimation of parameters required by the controllers, avoiding extra conversion calculations.

In this work, we will start in section 2 with the presentation of the nonlinear model of the $L S$, as well as the controllers used to reduce its nonlinear behavior in section 3. Section 4 is devoted to the estimation of the parameters, whereas section 5 deals with the adaptive algorithms used. In section 6 we present simulation results and conclusions are reserved for section 7 .

\section{THE LOUDSPEAKER MODEL}

The large signal behavior of an electrodynamic loudspeaker can be modeled by a pair of simplified nonlinear differential equations $[1,3]$ :

$$
\begin{gathered}
u=R_{e} i+\frac{d(L(x) i)}{d t}+B l(x) v \\
B l(x) i=m a+R v+k(x) x-\frac{1}{2} \frac{d L(x)}{d x} i^{2}
\end{gathered}
$$

The equation 1 describes the electrical part of the loudspeaker, where $u$ is the voltage supplied to the loudspeaker, $i$ is the current and $v$ is the loudspeaker diaphragm velocity. The main electrical elements are the voice-coil electrical resistance $R_{e}$ and the voice-coil nonlinear self inductance $L(x)$. For the mechanical part, equation 2 has $x$ as the diaphragm displacement and $a$ as its acceleration. Actually, this equation represents a damped massspring mechanical system with a moving mass $m$, a mechanical damping $R$ and a nonlinear stiffness $k(x)$. There is also an added reluctance force $-\frac{1}{2} \frac{d L(x)}{d x} i^{2}$ caused by the nonlinear selfinductance. Both parts are interconnected by the $L S$ motor causing interdependence between the two equations and the ratio between the force produced by the motor and the current is the nonlinear force-factor $B l(x)$. All the nonlinearities present in the model are displacement-dependent and can be approximated by $n$-th order polynomials:

$$
\begin{aligned}
B l(x) & =b l_{0}+b l_{1} x+b l_{2} x^{2}+\cdots+b l_{n} x^{n} \\
L(x) & =l_{0}+l_{1} x+l_{2} x^{2}+\cdots+l_{n} x^{n} \\
k(x) & =k_{0}+k_{1} x+k_{2} x^{2}+\cdots+k_{n} x^{n}
\end{aligned}
$$

So, the model of the $L S$ includes a set of linear parameters, $\left(R_{e}\right.$, $m$ and $R$ ) plus the several polynomial terms $b l_{n}, l_{n}$ and $k_{n}$.

\section{LOUDSPEAKER CONTROLLERS PARAMETERS}

Although theory shows [3] that current drive (using high output impedance amplifiers) is more suitable for the control of the $L S$ 
than voltage drive (using low output impedance amplifiers), commercially the latter is still preferred. So, two types of controllers will be considered.

\subsection{Current drive controller}

A current drive controller can be written as [3]

$$
\begin{aligned}
i= & G^{\prime} Q(x) i^{\prime}+\left[R(x)-\frac{\omega_{p}^{\prime}}{Q_{p}^{\prime}} Q(x)\right] v+ \\
& +\left[S(x)-\omega_{p}^{\prime 2} Q(x)\right] x+T(x) i^{* 2}
\end{aligned}
$$

where $i^{\prime}$ is the controller input, $i$ is the current to feed the loudspeaker and $i^{*}$ is the previous value of the loudspeaker current. The resonance frequency $\omega_{p}^{\prime}$, the quality factor $Q_{p}^{\prime}$ and the gain $G^{\prime}$ define the linear frequency response of the controlled loudspeaker. The nonlinear functions $Q(x), R(x), S(x)$ and $T(x)$ are approximated by polynomials:

$$
\begin{aligned}
& Q(x) \quad=\frac{m}{B l(x)} \quad=q_{0}+q_{1} x+q_{2} x^{2}+\cdots \\
& R(x) \quad=\frac{R}{B l(x)} \quad=r_{0}+r_{1} x+r_{2} x^{2}+\cdots \\
& S(x) \quad=\frac{k(x)}{B l(x)} \quad=s_{0}+s_{1} x+s_{2} x^{2}+\cdots \\
& T(x)=-\frac{1}{2 B l(x)} \frac{d L(x)}{d x}=t_{0}+t_{1} x+t_{2} x^{2}+\cdots
\end{aligned}
$$

Equations 7 to 10 contain all the unknown loudspeaker parameters needed to be determined for this controller.

\subsection{Voltage drive controller}

A voltage drive controller can be written as [3]

$$
\begin{aligned}
u= & R_{e} G^{\prime} Q(x) u^{\prime}+ \\
& +\left[R_{e} R(x)+B l(x)-R_{e} \frac{\omega_{p}^{\prime}}{Q_{p}^{\prime}} Q(x)\right] v+ \\
& +\left[R_{e} S(x)-R_{e} \omega_{p}^{\prime 2} Q(x)\right] x+T(x) i^{2}+ \\
& +\frac{d(L(x) i)}{d t}-\frac{R_{e}^{2}}{\omega_{L}^{\prime}} G^{\prime} Q(x) \frac{d i^{\prime}}{d t}
\end{aligned}
$$

where the controller input is now $u^{\prime}$. For specifying the controlled frequency response, an additional parameter $\left(\omega_{L}^{\prime}\right)$ has to be defined because a voltage driven loudspeaker has a larger order (third) than current driven. The signal $i^{\prime}$, for this controller, is a virtual current corresponding to the current the loudspeaker wold have if it was linear and had a frequency response defined by $G^{\prime}, \omega_{p}^{\prime}, Q_{p}^{\prime}$ e $\omega_{L}^{\prime}$.

$$
i^{\prime}=\frac{1}{G^{\prime}} a+\frac{\omega_{p}^{\prime}}{G^{\prime} Q_{p}^{\prime}} v+\frac{\omega_{p}^{\prime 2}}{G^{\prime}} x
$$

The unknown parameters of the $L S$ required for this controller are the same $(Q(x), R(x), S(x)$ and $T(x)$ coefficients) as before in addition to $R_{e}$ and the coefficients of $B l(x)$ and $L(x)$.

\section{PARAMETER ESTIMATION}

The next phase is the determination of estimation systems that can obtain values for the parameters defined above. Figure 1 represents a topology, known as parallel plant modeling, where the plant (the loudspeaker) is placed in parallel with a model. The output of the

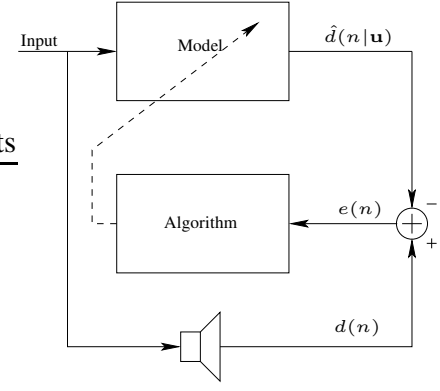

Fig. 1. Diagram of the adaptive system.

plant $d(n)$ is the desired response, the output of the model is an estimate $\hat{d}(n \mid \mathbf{u})$ of the desired response an the difference between them

$$
e(n)=d(n)-\hat{d}\left(n \mid \mathbf{u}_{n}\right)
$$

is the estimation error. When the estimate is close to the desired response then the estimation error is small and the model is said to be adapted to the plant. The adaptive algorithm tries to find a parameter vector $\mathbf{w}$ that minimizes a cost function $J(\mathbf{w})$, namely the Wiener filters [6] use the mean-squared error

$$
J(\mathbf{w})=\left[e(n) e^{*}(n)\right] .
$$

But before going more into to the algorithms themselves, let us define the models to be used to measure de loudspeaker parameters required by the controllers.

Starting by the current drive controller, we can see the resemblance between the functions $Q(x), R(x), S(x)$ and $T(x)$ (equations 7 to 10$)$ of the controller and the terms appearing in equation 2. In fact, if we solve equation 2 in respect to $i$, we get the following equation

$$
i=Q(x) a+R(x) v+S(x) x+T(x) i^{2}
$$

which can be used as the model, using the current $i$ as an estimate of the loudspeaker response $(\hat{d}(n \mid \mathbf{u})=i)$, of course being compared with the current of the real loudspeaker. Note that the current drive control does not require any other parameters other than those given by the model of equation 15 , meaning that current drive control only requires the estimation of the mechanical part parameters, and none from the electrical part.

The equation 15, however, poses a problem because it is nonlinear, and the model part of an adaptive filter usually [6] is a finite impulse response (FIR) filter, which is described by the expression $\hat{d}(n)=\mathbf{w} \mathbf{u}$, where $\hat{d}(n)$ is the filter desired output, $\mathbf{u}$ is a tap-delay vector of the input

$$
\mathbf{u}=[u(n), u(n-1), u(n-2), \cdots]^{T}
$$

and $\mathbf{w}$ is a vector containing the weights for each filter tap. This kind of filter is linear and is expected to be adapted to the response of a linear system.

However, if we take equation 15 and expand all it's polynomials we get

$$
\begin{aligned}
i^{\prime}= & q_{0} \frac{d^{2} x}{d t^{2}}+q_{1} x \frac{d^{2} x}{d t^{2}}+q_{2} x^{2} \frac{d^{2} x}{d t^{2}}+\cdots+r_{0} \frac{d x}{d t}+ \\
& +r_{1} x \frac{d x}{d t}+r_{2} x^{2} \frac{d x}{d t}+\cdots+s_{0} x+s_{1} x^{2}+ \\
& +s_{2} x^{3}+\cdots+t_{0} i^{2}+t_{1} x i^{2}+t_{2} x^{2} i^{2}+\cdots,
\end{aligned}
$$




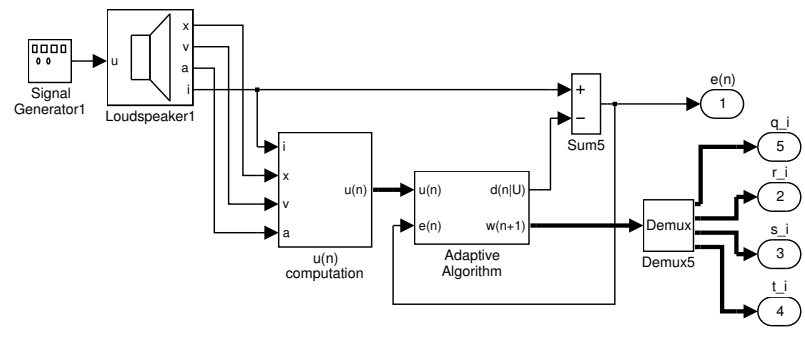

Fig. 2. The estimation system for the mechanical part

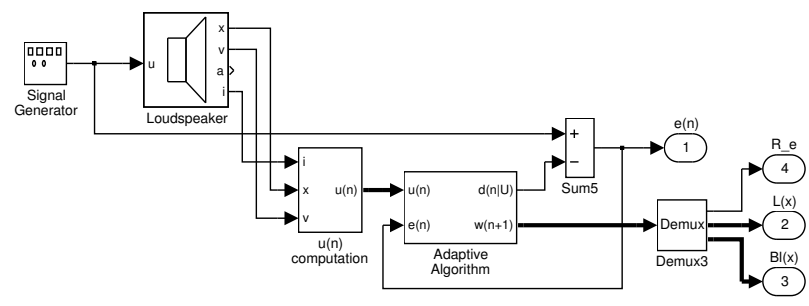

Fig. 3. The estimation system for the electrical part

which can be written as $i^{\prime}=\mathbf{w u}$ if we make

$$
\mathbf{w}=\left[q_{0}, q_{1}, q_{2}, \ldots, r_{0}, r_{1}, r_{2}, \ldots, s_{0}, s_{1}, s_{2}, \ldots, t_{0}, t_{1}, t_{2}, \ldots\right]
$$

as the parameter vector to be determined and

$$
\begin{aligned}
\mathbf{u}= & {\left[\frac{d^{2} x}{d t^{2}}, x \frac{d^{2} x}{d t^{2}}, x^{2} \frac{d^{2} x}{d t^{2}}, \cdots, \frac{d x}{d t}, x \frac{d x}{d t}, x^{2} \frac{d x}{d t}, \cdots\right.} \\
& \left.\cdots, x, x^{2}, x^{3}, \cdots, i^{2}, x i^{2}, x^{2} i^{2}, \cdots\right]^{T}
\end{aligned}
$$

as an input vector containing nonlinear state signals from the loudspeaker. So, we manage to get a formalization of the model similar to the one used for a FIR filter but, instead of a tap-delay vector, we now have a nonlinear state input vector $\mathbf{u}$. Then we can apply the same algorithms used in adaptive filters, namely the Kalman and $R L S$ algorithms.

For the voltage drive controller, the extra required parameters are already in equation 1 that can be directly used as a model for the adaptive algorithms. In this case, by means of expanding polynomials as was done for current drive, we get

$$
\mathbf{w}=\left[R_{e}, l_{0}, l_{1}, l_{2}, \ldots, b l_{0}, b l_{1}, b l_{2}, \ldots\right]
$$

for the parameter vector and

$$
\mathbf{u}=\left[i, \frac{d}{d t} i, \frac{d}{d t}(x i), \frac{d}{d t}\left(x^{2} i\right), \cdots, \frac{d x}{d t}, x \frac{d x}{d t}, x^{2} \frac{d x}{d t}, \cdots\right]_{(21)}^{T}
$$

for the input vector. This controller requires estimation of both mechanical and electrical part parameters, so it requires the use of the two estimation systems simultaneously.

Note that not all the adaptive filter theory results [6] remain valid after such changes as we have done, namely the convergence conditions of the algorithms no longer apply.

\section{ADAPTIVE ALGORITHMS}

It was already mentioned that the Wiener filters use the meansquared error as a cost function. To update the parameters vector $\mathbf{w}$, the steepest-descent algorithm uses the recursive relation

$$
\mathbf{w}(n+1)=\mathbf{w}(n)+\frac{1}{2} \mu[-\nabla(n)]
$$

where $\nabla(n)=\frac{d J(n)}{d \mathbf{w}(n)}$ is the gradient of the cost function $J(\mathbf{w})$ in relation to the parameter vector $\mathbf{w}$. But the problem with this algorithm is the requirement of knowing the input vector correlation matrix and the cross correlation vector between the input vector $\mathbf{u}$ and the desired response $d(n)$, both unfortunately unknown. So, different algorithms, sub-optimal, are used instead, from which we are going to use the Kalman algorithm and the $R L S$ algorithm.

The reader who needs a more in-depth discussion about adaptive algorithms, can find it in [6]. We are going to limit ourselves to a very superficial description, just enough to alow an implementation of the algorithms.

\subsection{Kalman Algorithm}

The Kalman algorithm makes a number of computations, in each iteration, described by the following set of expressions:

$$
\begin{aligned}
& \mathbf{g}(n)= \mathbf{K}(n, n-1) \mathbf{u}(n)\left[J_{\text {min }}+\right. \\
&\left.+\mathbf{u}^{T}(n) \mathbf{K}(n, n-1) \mathbf{u}(n)\right]^{-1} \\
& e(n)= d(n)-\mathbf{u}^{T}(n) \hat{\mathbf{w}}(n-1) \\
& \hat{\mathbf{w}}(n)=\hat{\mathbf{w}}(n-1)+\mathbf{g}(n) e(n) \\
& \mathbf{K}(n)=\mathbf{K}(n, n-1)- \\
&-\mathbf{g}(n) \mathbf{u}^{T}(n) \mathbf{K}(n, n-1) \\
& \mathbf{K}(n+1, n)=\mathbf{K}(n)+q \mathbf{I}
\end{aligned}
$$

The value of the constant Jmin should be about 0.001 to 0.01 times the desired response $d(n)$ variance. The initial conditions are $\mathbf{w}(0)=\mathbf{0}$ and $\mathbf{K}(0)=c \mathbf{I}$, where $c$ is a positive constant and $\mathrm{I}$ is the identity matrix.

\subsection{RLS Algorithm}

The $R L S$ algorithm also uses a series of computations for each iteration, but now using the expressions

$$
\begin{aligned}
\mathbf{k}(n) & =\frac{\lambda^{-1} \mathbf{P}(n-1) \mathbf{u}(n)}{1+\lambda^{-1} \mathbf{u}^{T}(n) \mathbf{P}(n-1) \mathbf{u}(n)} \\
e(n) & =d(n)-\hat{\mathbf{w}}^{T}(n-1) \mathbf{u}(n) \\
\hat{\mathbf{w}}(n) & =\hat{\mathbf{w}}(n-1)+\mathbf{k}(n) e(n) \\
\mathbf{P}(n) & =\lambda^{-1} \mathbf{P}(n-1)-\lambda^{-1} \mathbf{k}(n) \mathbf{u}^{T}(n) \mathbf{P}(n-1)
\end{aligned}
$$

being initialized with $\mathbf{P}(0)=\delta^{-1} \mathbf{I}$ and $\mathbf{w}(0)=\mathbf{0}$, where $\delta$ is a small positive constant of no important influence if the number of iterations is large.

\section{SIMULATIONS}

The two loudspeaker parameter estimation systems, as shown in figures 2 and 3, where simulated in the SIMULINK $\mathbb{1}{ }^{1}$ environment, using a loudspeaker simulation model, with the parameters

\footnotetext{
${ }^{1}$ The MathWorks, Inc.
} 


\begin{tabular}{|l|ccccc|}
\cline { 2 - 6 } \multicolumn{1}{c|}{} & order & & & & \\
\hline Parameter & 0 & 1 & 2 & 3 & 4 \\
\hline$B l(T m)$ & 9,06 & 566 & $-1,52 e 5$ & $-1,90 e 6$ & $2,72 e 9$ \\
$k\left(N m^{-1}\right)$ & $2,64 e 3$ & $1,39 e 5$ & $6,94 e 7$ & 0 & 0 \\
$L(H)$ & $1,62 e-3$ & 0.111 & -17.82 & -444 & $8,88 e 4$ \\
\hline$R\left(N m^{-2}\right)$ & 0,8 & & & & \\
$m(K g)$ & 0,036 & & & & \\
$R_{e}(\Omega)$ & 5,4 & & & & \\
\hline
\end{tabular}

Table 1. Loudspeaker parameters used in simulations

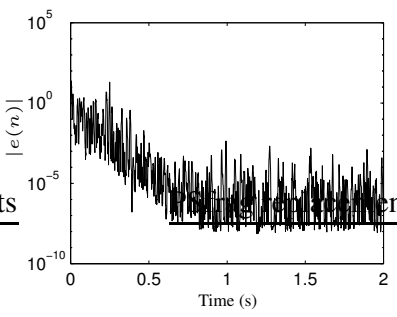

(a) Kalman, mechanical

(c) Kalman, electrical

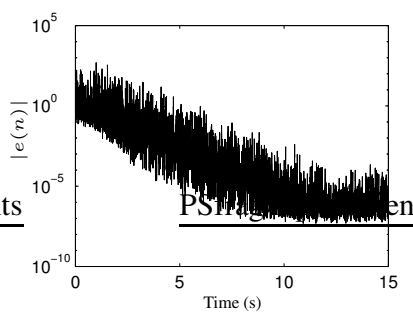

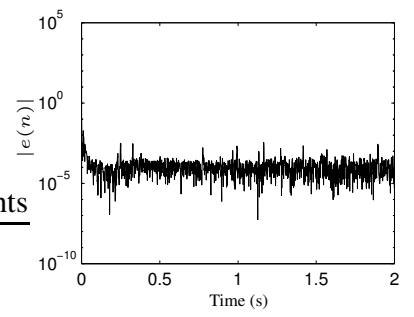

(b) $R L S$, mechanical

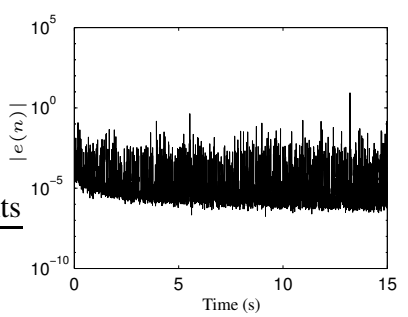

(d) $R L S$, electrical
Fig. 4. Time evolution of the modulus of the estimation error for the Kalman and the RLS mechanical and electrical loudspeaker parameter estimation systems.

presented in table 1, as the loudspeaker whose parameters are to be determined. Both algorithms in both mechanical and electrical part estimation systems where able to converge, being evolution of the absolute value of the estimation error presented in figure 4. As seen above, the estimation error is the difference between the loudspeaker (or desired) response and the model (included in the estimation system) response (or estimated response), so the smaller its absolute value the better the estimation is, ideally should be zero.

For the mechanical part, the Kalman algorithm reaches the minimum error level at $10^{-5}$ in about 0.8 seconds while the $R L S$ algorithm reaches a $10^{-4}$ error level in 0.2 seconds. Thus, the $R L S$ algorithm is faster at the expense of an higher error level. The same kind of behavior can be seen for the electrical part but at a slower rate, taking both algorithms about 15 times longer to reach the minimum estimation error levels. The Kalman algorithm reaches $10^{-5}$ in 12 seconds and the $R L S$ algorithm reaches $10^{-3}$ in 3 seconds.

\section{CONCLUSION}

Loudspeakers $(L S)$ have non-ideal frequency response and, because they are highly nonlinear transducers, produce harmonic distortion. In order to increase their output at low frequencies, large enclosures have to be used causing practical and usability problems. Also, limitations on enclosure size means a smaller volume of air inside the enclosure causing, due to the highly nonlinear behavior of air compression, additional distortion. To further reduce the distortion, nonlinear controllers are used requiring a large set of parameters well tuned to the controlled $L S$. Due to several causes, these parameters are not know and do not remain constant, thus their accurate determination (even in normal operation), without the use of special signal or special $L S$ conditioning, is mandatory to the good performance of the controller.

In this work, a simplified model of the loudspeaker was presented, as well as nonlinear controllers used to reduce its nonlinear behavior. The model was presented in such a way that the determined parameter could be directly used by the controllers, avoiding extra conversion calculations, and was concluded that the current drive controller only requires the estimation of the parameters of the mechanical part while the voltage drive requires both, the mechanical and the electrical. So, based on the model and in some modifications done to the classical adaptive filter in order to apply it to nonlinear systems, two systems able to estimate these parameters from the $L S$ where developed, one for the mechanical part and the other for the electrical. These systems can use classical adaptive algorithms, in particular, the Kalman and the $R L S$ presented in this work.

Due to the modifications done, not all the results from the adaptive filter theory remain valid, namely the convergence con-

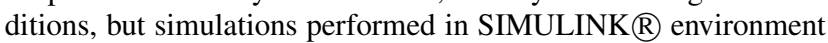
showed that the two algorithms converge in the two systems, although the electrical parameter estimation system was about 15 times slower than the mechanical. The comparison of the two adaptive algorithms revealed a tradeoff between the lower estimation error attained by the Kalman algorithm and the faster convergence of the $R L S$ algorithm for both systems.

\section{REFERENCES}

[1] Arie J. M. Kaizer, "Modeling of the nonlinear response of an electrodynamic loudspeaker by a volterra series expansion," $J$. Audio Eng. Soc., vol. 35, no. 6, pp. 421-433, June 1987.

[2] Wolfgang Klippel, "Nonlinear large-signal behavior of electrodynamic loudspeakers at low frequencies," J. Audio Eng. Soc., vol. 37, no. 5, pp. 353-362, Mai 1989.

[3] Ricardo Adriano Ribeiro, Controlador da Resposta de Altifalantes de Graves, MSC Thesis (in Portuguese), 2004.

[4] Jeffrey Harrison, "An integral limitation upon loudspeaker frequency response for a given enclosure volume," J. Audio Eng. Soc., vol. 44, no. 12, pp. 1097-1103, December 1996.

[5] Wolfgang Klippel, "The mirror filter-a new basis for reducing nonlinear distortion and equalizing response in woofer systems," J. Audio Eng. Soc., vol. 40, pp. 675-691, September 1992.

[6] Simon Haykin, Adaptive Filter Theory, Prentice-Hall, 2001. 